\title{
Curve Tracking Control for Legged Locomotion
}

\author{
Fumin Zhang \\ Department of Mechanical and Aerospace Engineering, Princeton University \\ fzhang@princeton.edu
}

\begin{abstract}
Recent developments in legged mobile robots call for curve tracking control laws that enable safe movements in complex environment. The lateral leg spring (LLS) model captures planar locomotion dynamics that are platform independent. We derive a hybrid feedback control law for the LLS model so that the center of mass of a legged runner follows a curved path in horizontal plane. The control law enables the runner to change the placement and the elasticity of its legs to move in a desired direction. Stable motion along a curved path is achieved using curvature, bearing and relative distance between the runner and the curve as feedback. Constraints on leg parameters determine the class of curves that can be followed. These results are applicable to a wide range of legged robots.
\end{abstract}

\section{INTRODUCTION}

From tiny ants to large elephants, legged locomotion is the dominant method that animals use to move on the ground. Although the leg structures are vastly different across species, the mechanisms for walking, jumping or running obey strikingly similar principles. These similarities are captured by mathematical models such as the spring-loaded inverted pendulum (SLIP) model, see [1], and the lateral leg spring (LLS) model in [2]-[5]. The LLS model describes motion in the horizontal plane and the SLIP model describes motion in the sagittal (vertical) plane.

We use the LLS model in this paper in designing curve tracking control in the horizontal plane; the runner is modeled as a rigid body with two weightless springs attached to a point in the body called the center of pressure (COP). Each spring represents legs on one side of the body. The COP is usually not coincident with the center of mass (COM). Legged locomotions can be self-stabilized-the running or walking gaits stay close to being periodic under disturbances-without feedback control. As indicated by a recent review [6], the self-stabilized walking and running happen when the runner moves along a straight line.

There are many interests in engineering practice to design and build legged robots that are versatile on rough terrains. Legged robots are greatly appreciated in applications such as searching and rescuing missions and planet exploration. In most missions, the robots must be able to move along an arbitrary path. Feedback control is needed for tracking curved path as well as stabilizing the periodic gaits.

In this paper, we develop feedback control law for the LLS model so that the COM follows a curved path. The

This work was supported in part by ONR grants N00014-02-10826, N00014-02-1-0861 and N00014-04-1-0534. I want to thank Philip Holmes, Justin Seipel and Naomi Leonard for discussions and suggestions. legged locomotion modeled by the LLS model is a hybrid system. Correspondingly, the tracking control contains a discrete tracking algorithm which guarantees convergence to the desired curve and a continuous law to control the leg parameters of the LLS model. Section II serves as an introduction to the LLS model. The discrete tracking algorithm is developed in Section III. In Section IV, control laws are developed for the leg parameters to enable the discrete tracking algorithm. The constraints on the parameters and the effects on the tracking behavior are discussed in Section V. We provide simulation results in Section VI.

\section{MOTION OF THE COM}

In the horizontal plane, motion generated by the LLS model starts when the free end of one spring (or leg) is placed at a touchdown point $P$. At this starting moment, the spring is at its free length $\eta_{0}$. If the COM has a non-zero initial velocity $\mathbf{v}$ that is not perpendicular to the spring, then the spring will be first compressed to a minimum length and later be restored to its free length. This process, starting and ending with the spring at its free length $\eta_{0}$, is called a stance phase or simply a stance. The COM moves from the starting position to an ending position after a stance. Suppose that mechanical energy is conserved during each stance, then the starting and ending speed of a stance is identical. As shown in Figure 1, the end of one stance serves as the beginning of the next stance with the touchdown point $P$ shifted from one side to the other. This allows us to distinguish left stances from right stances based on which leg is supporting the body.

The rigid body moves forward as a result of switching between left and right stances. As shown in Figure 1, we use $\mathbf{r}_{i}, i=1,2, \ldots$, to denote the position of the COM in a lab fixed coordinate frame at the beginning of the $i$ th stance. We use $\alpha_{i}$ to denote the angle between the velocity $\mathbf{v}_{i}$ and the spring at rest. For a right stance, the angle is measured counter-clockwise from the spring to the velocity vector. For a left stance, the angle is measured clockwise from the spring to the velocity vector. Under this convention for measuring angles, $\alpha_{i}$ has to be within the interval $(0, \pi / 2)$ to generate forward locomotion.

We can view $\mathbf{r}_{i}$ as points on a curve $\Gamma$ which is formed by straight line segments that connects $\mathbf{r}_{i-1}$ with $\mathbf{r}_{i}$ for all $i$. This curve $\Gamma$ is not the actual trajectory of the COM, but it intersects with the trajectory of the COM at the points $\mathbf{r}_{i}$. We then let $\mathbf{q}_{i}=\mathbf{r}_{i+1}-\mathbf{r}_{i}$ and define $q_{i}=\left\|\mathbf{q}_{i}\right\|$. We also define an angle $\delta_{i}$ as the angle between the vector $\mathbf{q}_{i}$ 


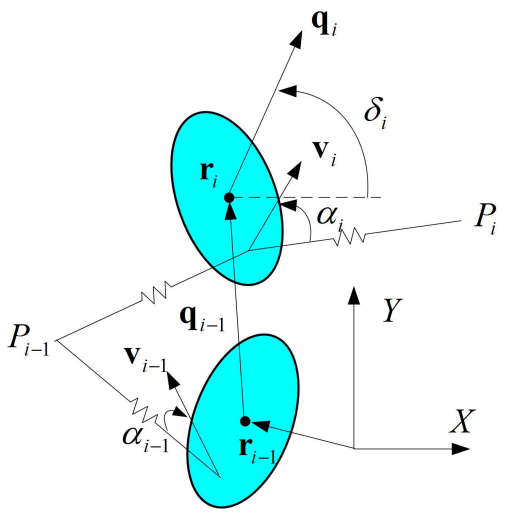

Fig. 1. The LLS model for legged locomotion. One left stance followed by one right stance are plotted. The center of mass (COM) is translated from $\mathbf{r}_{i-1}$ to $\mathbf{r}_{i}$. The velocities of the COM at the beginning of each stance are the vectors $\mathbf{v}_{i-1}$ and $\mathbf{v}_{i}$. The angle between $\mathbf{v}_{i}$ and the spring (leg) is $\alpha_{i}$. The angle between vector $\mathbf{q}_{i}$ and the horizontal axis $X$ is $\delta_{i}$. The positive directions for all angles are as shown.

and the horizontal axis of the lab frame, measured counterclockwise from the axis. This angle describes the direction of curve $\Gamma$ for the $i$ th stance. The motion of the COM can now be described by a discrete system

$$
\mathbf{r}_{i+1}=\mathbf{r}_{i}+\left[q_{i} \cos \delta_{i}, q_{i} \sin \delta_{i}\right]^{T} .
$$

\section{TRACKING A DETECTED BOUNDARY}

Suppose at the position $\mathbf{r}_{i}$, the runner is able to detect a segment of a boundary curve from sensor information, c.f., [7] and [8]. Suppose the runner is also able to estimate a point on the boundary curve that has the minimum distance to the COM. We call this point the closest point $\mathbf{r}_{\mathrm{c}_{i}}$ shown in Figure 2. By selecting two extra points on the boundary near $\mathbf{r}_{\mathrm{c}_{i}}$, the runner can estimate the tangent vector to the boundary curve $\mathbf{x}_{\mathrm{c}_{i}}$ and the curvature of the boundary curve $\kappa_{i}$ using algorithms summarized in [7]. Here we suppose all estimates are perfect.

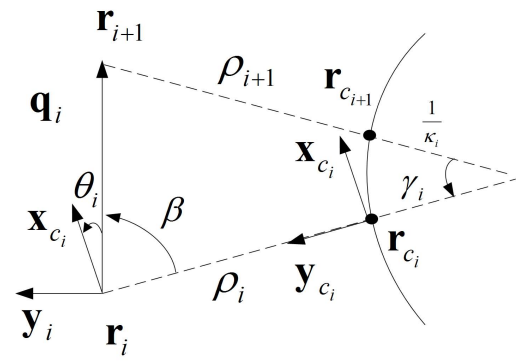

Fig. 2. The movement of the COM near a boundary curve. $\theta_{i}$ is the angle between $\mathbf{q}_{i}$ and $\mathbf{x}_{\mathrm{C}_{i}} . \rho_{i}$ is the distance between the COM and the closest point. $\kappa_{i}$ is the curvature of the curve. $\gamma_{i}$ is the center angle of the arc connecting $\mathbf{r}_{\mathrm{C}_{i}}$ and $\mathbf{r}_{\mathrm{c}_{i+1}}$

We let $\rho_{i}$ be the distance between the COM at $\mathbf{r}_{i}$ and the closest point at $\mathbf{r}_{\mathrm{c}_{i}}$. We also let $\mathbf{x}_{i}=\frac{\mathbf{q}_{i}}{\left\|\mathbf{q}_{i}\right\|}$ be the unit vector in the direction of $\mathbf{q}_{i}$. We can then define two right handed frames-one at $\mathbf{r}_{i}$ and the other at $\mathbf{r}_{\mathrm{c}_{i}}$-by defining two unit normal vectors $\mathbf{y}_{i}$ and $\mathbf{y}_{\mathrm{c}_{i}}$ as shown in Figure 2. The angle $\theta_{i}$ between $\mathbf{x}_{i}$ and $\mathbf{x}_{\mathrm{c}_{i}}$ is defined by letting

$$
\cos \theta_{i}=\mathbf{x}_{i} \cdot \mathbf{x}_{\mathrm{c}_{i}} \text { and } \sin \theta_{i}=-\mathbf{x}_{i} \cdot \mathbf{y}_{\mathrm{c}_{i}} .
$$

In Figure 2, the closest point on the boundary moves from $\mathbf{r}_{\mathrm{c}_{i}}$ to $\mathbf{r}_{\mathrm{c}_{i+1}}$ during a stance. We then assume that each stance is short enough so that the curvature of the boundary curve detected during this period can be approximated by a finite constant $\kappa_{i}$. From Figure 2, we observe $\beta=\frac{\pi}{2}-\theta_{i}$. Therefore, according to the cosine law, we have

$$
\left(\rho_{i+1}+\frac{1}{\kappa_{i}}\right)^{2}=\left(\rho_{i}+\frac{1}{\kappa_{i}}\right)^{2}-2\left(\rho_{i}+\frac{1}{\kappa_{i}}\right) q_{i} \sin \theta_{i}+q_{i}^{2} .
$$

We can view $\rho_{i}$ as state variables, consider $q_{i}$ as the control over step size, and $\theta_{i}$ as steering control. Equation (3) describes the controlled relative motion between the COM and the detected boundary curve.

Suppose that the step size $q_{i}$ has been determined. We need to find $\theta_{i}$ such that $\rho_{i}$ converges to a desired value $\rho_{\mathrm{c}}$ as $i \rightarrow \infty$. If we can find a feedback control law $f_{i}(\cdot)$ such that

$$
\rho_{i+1}-\rho_{i}=f_{i}\left(\rho_{i}-\rho_{\mathrm{c}}\right),
$$

then we can choose the function $f_{i}(\cdot)$ so that $\rho_{i}-\rho_{\mathrm{c}} \rightarrow 0$. For example, we may let

$$
f_{i}\left(\rho_{i}-\rho_{\mathrm{c}}\right)=-K_{i} \cdot\left(\rho_{i}-\rho_{\mathrm{c}}\right)
$$

where $0<K_{i}<1$. Observe that

$$
\rho_{i+1}-\rho_{i}=\rho_{i+1}-\rho_{\mathrm{c}}-\left(\rho_{i}-\rho_{\mathrm{c}}\right),
$$

and this leads to

$$
\rho_{i+1}-\rho_{\mathrm{c}}=\left(1-K_{i}\right)\left(\rho_{i}-\rho_{\mathrm{c}}\right) .
$$

Since $-1<1-K_{i}<1$, it is true that $\rho_{i} \rightarrow \rho_{\mathrm{c}}$ as $i \rightarrow \infty$.

We now define $\lambda_{i}=\rho_{i}+\frac{1}{\kappa_{i}}$. In order to achieve (4), we replace $\left(\rho_{i+1}+\frac{1}{\kappa_{i}}\right)$ with $\left(\lambda_{i}+f_{i}\right)$ in (3). From (3), we can solve for $\sin \theta_{i}$ as

$$
\begin{aligned}
\sin \theta_{i} & =\frac{-f_{i}^{2}-2 \lambda_{i} f_{i}+q_{i}^{2}}{2 \lambda_{i} q_{i}} \\
& =\frac{q_{i}}{2 \lambda_{i}}-\frac{f_{i}}{q_{i}}-\frac{f_{i}^{2}}{2 \lambda_{i} q_{i}} .
\end{aligned}
$$

When $f_{i}=0$, the solution is $\theta_{i}=\sin ^{-1}\left(q_{i} / 2 \lambda_{i}\right)$. This corresponds to the runner running parallel to the boundary curve.

Note that when $\kappa_{i} \rightarrow 0$, then $\lambda_{i} \rightarrow \infty$. The limit of equation (8) is $\sin \theta_{i}=-\frac{f_{i}}{q_{i}}$, which is equivalent to $\rho_{i+1}-$ $\rho_{i}=-q_{i} \sin \theta_{i}$. This equation indeed describes the relative motion between the COM and a straight line. Therefore, all the results that will be obtained from (8) are applicable to straight lines by letting $\lambda_{i} \rightarrow \infty$.

Lemma 3.1: A solution exists for $\theta_{i}$ in equation (8) if and only if $f_{i} \in\left[-\left(2 \lambda_{i}+q_{i}\right), \min \left\{-q_{i}, q_{i}-2 \lambda_{i}\right\}\right]$ or $f_{i} \in\left[\max \left\{-q_{i}, q_{i}-2 \lambda_{i}\right\}, q_{i}\right]$.

Proof: A solution exists for $\theta_{i}$ if and only if

$$
\frac{-f_{i}^{2}-2 \lambda_{i} f_{i}+q_{i}^{2}}{2 \lambda_{i} q_{i}} \leq 1
$$


and

$$
\frac{-f_{i}^{2}-2 \lambda_{i} f_{i}+q_{i}^{2}}{2 \lambda_{i} q_{i}} \geq-1 .
$$

One can verify that these are equivalent to

$$
-\left(2 \lambda_{i}+q_{i}\right) \leq f_{i} \leq \min \left\{-q_{i}, q_{i}-2 \lambda_{i}\right\}
$$

or

$$
\max \left\{-q_{i}, q_{i}-2 \lambda_{i}\right\} \leq f_{i} \leq q_{i}
$$

Lemma 3.2: Suppose $0<q_{i}<2 \lambda_{i}$. Let $f_{i}=-K_{i}\left(\rho_{i}-\right.$ $\left.\rho_{\mathrm{c}}\right)$. There exists $K_{i} \in(0,2)$ such that (12) is satisfied and $\theta_{i}$ in (8) has a solution.

Proof: If $\left|\rho_{i}-\rho_{\mathrm{c}}\right|<\frac{1}{2} \min \left\{q_{i}, 2 \lambda_{i}-q_{i}\right\}$, we may let $K_{i}$ be any value in the interval $(0,2)$ and (12) is satisfied. If $\left|\rho_{i}-\rho_{\mathrm{c}}\right| \geq \frac{1}{2} \min \left\{q_{i}, 2 \lambda_{i}-q_{i}\right\}$, we may let

$$
0<K_{i}<\min \left\{2, \frac{q_{i}}{\left|\rho_{i}-\rho_{\mathrm{c}}\right|}, \frac{2 \lambda_{i}-q_{i}}{\left|\rho_{i}-\rho_{\mathrm{c}}\right|}\right\}
$$

which satisfies (12).

The condition $q_{i}<2 \lambda_{i}$ in Lemma 3.2 indicates that each step size should not be too big. The following theorem is a direct consequence of Lemma 3.2.

Theorem 3.3: Suppose the step size $q_{i}$ satisfy $0<q_{i}<$ $2 \lambda_{i}$. Let $f_{i}=-K_{i}\left(\rho_{i}-\rho_{\mathrm{c}}\right)$. Select $K_{i} \in(0,2)$ such that $\theta_{i}$ in (8) has a solution. Then under the control $q_{i}$ and $\theta_{i}$, we have $\rho_{i} \rightarrow \rho_{\mathrm{c}}$ as $i \rightarrow \infty$.

Once $\theta_{i}$ is determined from (8), we can compute the direction of $\mathbf{q}_{i}$ in the lab frame. This is because

$$
\delta_{i}=\zeta_{i}-\theta_{i}
$$

where $\zeta_{i}$ is the angle between the horizontal axis of the lab frame and the vector $\mathbf{x}_{c_{i}}$, measured counter clockwise from the horizontal axis. Knowing $q_{i}$ and $\delta_{i}$ allows us to compute the position of the COM for the next stance from the discrete system given by equation (1).

\section{Control The LLS MODEL}

In order to generate desired $q_{i}$ and $\delta_{i}$ to control the COM movement, the runner needs to change its leg placements or the elasticity of the legs. We investigate the dynamics during each stance to establish the relations between the COM motion and the leg parameters.

Leg parameters for a right stance and a left stance often differ only by the sign. Since a right stance is always followed by a left stance and vice versa, we use the convention that the stance $k$ is always a right stance and stance $k+1$ is always a left stance. Therefore, stance $k+2$ must be a right stance, etc. In the following, we will only show detailed derivation for a right stance; similar results for a left stance will be listed directly.

We set up a polar coordinate system at the touchdown point $P_{k}$ with the horizontal $\mathrm{x}$-axis parallel to the horizontal $X$-axis of the fixed lab frame. Let $(\eta, \psi)$ be the polar coordinates of the COM in this frame and let $\sigma$ describe the orientation of the rigid body. Then the total energy is

$$
E=\frac{1}{2} m \dot{\eta}^{2}+\frac{1}{2} m \eta^{2} \dot{\psi}^{2}+\frac{1}{2} I \dot{\sigma}^{2}+V(\eta)
$$

where we assume the spring has potential energy $V$ which depends only on its length.

During each stance, the dynamics can be described as a continuous nonlinear Hamiltonian system; the Hamilton equations are developed in [2]. The system is not integrable when the distance between the COM and the COP is nonzero. In this case numerical methods are necessary to compute the trajectory of the COM from knowledge of the states $(\eta, \psi, \sigma)$.

To illustrate analytical insights for the tracking problem, it is much easier to study the case when the COP and COM coincide. This is because the corresponding system is integrable. The Hamilton equations for the dynamics of the COM are

$$
\begin{aligned}
\dot{p}_{\eta} & =\frac{1}{m \eta^{3}} p_{\eta}^{2}-\frac{\partial V}{\partial \eta} \\
\dot{p}_{\psi} & =0
\end{aligned}
$$

where $p_{\eta}=m \dot{\eta}$ and $p_{\psi}=m \eta^{2} \dot{\psi}$.

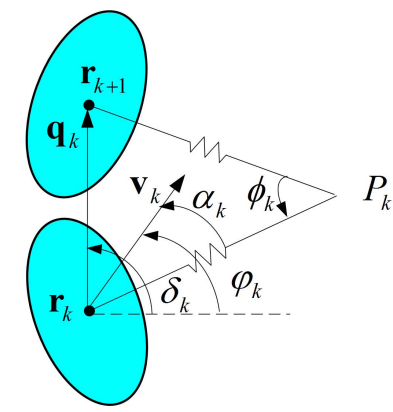

Fig. 3. The LLS model with COM coincide with COP. Angle $\phi_{k}$ is the center angle swept during the stance. Angle $\alpha_{k}$ is the angle between the leg and the velocity $\mathbf{v}_{k}$ at the moment of touch down.

We plot one right stance in Figure 3 . Let $\mathbf{v}_{k}$ be the velocity of the COM at the beginning of the $k$ th stance. This $\mathbf{v}_{k}$ provides initial value for the states $p_{\eta}$ and $p_{\psi}$ of the system (16). Because mechanical energy is conserved, the speed $v_{k}=\left\|\mathbf{v}_{k}\right\|$ satisfies $v_{k+1}=v_{k}$. We let $\alpha_{k}$ represent the angle measured from the leg to the velocity $\mathbf{v}_{k}$ at the moment of touchdown, and we call $\alpha_{k}$ the leg placement parameter. We use $\varphi_{k}$ to measure the direction of velocity in the lab frame. By using simple geometric relationships in Figure 3, we have

$$
\frac{\pi}{2}-\frac{\phi_{k}}{2}-\alpha_{k}=\delta_{k}-\varphi_{k} .
$$

Therefore, using equation (14),

$$
\zeta_{k}-\varphi_{k}-\theta_{k}=\frac{\pi}{2}-\alpha_{k}-\frac{\phi_{k}}{2} .
$$

Note that $\left(\zeta_{k}-\varphi_{k}\right)$ is the relative angle between $\mathbf{v}_{k}$ and $\mathbf{x}_{c_{k}}$ : the tangent vector to the boundary curve at the closest point. We know $\theta_{k}$ is the angle between vector $\mathbf{q}_{k}$ and the same tangent vector $\mathbf{x}_{c_{k}}$. Therefore, $\left(\zeta_{k}-\varphi_{k}-\theta_{k}\right)$ is the angle between $\mathbf{v}_{k}$ and $\mathbf{q}_{k}$. From (18), we see that changing $\alpha_{k}$ will change the direction of the COM movement $\mathbf{q}_{k}$. This is also true for a left stance where we have

$$
\zeta_{k+1}-\varphi_{k+1}-\theta_{k+1}=-\left(\frac{\pi}{2}-\alpha_{k+1}-\frac{\phi_{k+1}}{2}\right) .
$$


Another relationship we can derive from Figure 3 is

$$
q_{k}=2 \eta_{k} \sin \frac{\phi_{k}}{2}
$$

where $\eta_{k}$ represents the leg length at the moment of touchdown.

In (20) and (18), $q_{k}$ is the distance the runner wants to travel in one stance, the angle $\theta_{k}$ can be solved from (8), and the angle $\left(\zeta_{k}-\varphi_{k}\right)$ is known. We want to solve for the leg parameters $\alpha_{k}$ and $\eta_{k}$, but $\phi_{k}$ is still unknown. This unknown can be solved from the continuous system equations (16).

At the starting position of the $k$ th stance $\mathbf{r}_{k}$ and the ending position of the $k$ th stance $\mathbf{r}_{k+1}$, by conservation of the angular momentum, we have

$$
p_{\eta_{k+1}}=p_{\eta_{k}}=\eta_{k}^{2} \dot{\psi}=\eta_{k} v_{k} \sin \alpha_{k}
$$

Using the method of integration by quadrature, c.f. [9], we can compute the center swing angle $\phi_{k}$ for each stance as

$$
\phi_{k}=2 \int_{\eta_{k}}^{\eta_{\min }} \frac{\frac{p_{\eta_{k}}}{\eta^{2}}}{ \pm \sqrt{2 E-\frac{p_{\eta_{k}}^{2}}{\eta^{2}}-2 V(\eta)}} d \eta
$$

where $\eta_{\min }$ is the shortest length of the spring during the stance. When $\eta=\eta_{\text {min }}$, we have $\dot{\eta}=0$. Thus we can solve $\eta_{\min }$ from

$$
2 E-\frac{p_{\eta_{k}}^{2}}{\eta_{\min }^{2}}-2 V\left(\eta_{\min }\right)=0
$$

Since $\phi_{k}$ is now a known function of $\alpha_{k}, \eta_{k}$, and $b_{k}$, we can solve for any two of $\alpha_{k}, \eta_{k}$, and $b_{k}$ from (18) and (20) when keeping the other parameter constant. For a runner, controlling $\alpha_{k}$ means to find the appropriate angle between its leg and the direction of the COM motion. On the other hand, as reported by Jindrich and Full in [10], the cockroaches control the length $\eta_{k}$ by stretching or compressing their legs when turning. We see that changing $\eta_{k}$ will affect both $p_{\psi_{k}}$ and $V(\eta)$. This changes $\phi_{k}$ and hence controls $\left(\zeta_{k}-\varphi_{k}-\theta_{k}\right)$. Another means of steering is to change the potential energy $V(\eta)$,e.g., change the spring constant $b_{k}$, which also controls $\phi_{k}$.

If the conditions in Theorem 3.3 are satisfied, equation (8) always has a solution for $\theta_{i}$. The equations (18), (20) and (22) can be solved to implement the control $\theta_{i}$. Note that finding solutions for $\alpha_{k}$ and $\eta_{k}$ often requires numerical methods because $\phi_{k}$ is not a simple function of $\alpha_{k}$ and $\eta_{k}$.

\section{Tracking Behavior Under Constraints}

For every stance, the LLS model generates the COM movement $\mathbf{q}_{k}$ by controlling parameters such as $\alpha_{k}, \eta_{k}$ and $b_{k}$. In practice, these parameters all have to be bounded. These bounds post constraints on the possible $\mathbf{q}_{k}$ that can be generated by the LLS model. In this section, we discuss the constrained COM movement and investigate the constrained tracking behavior when a runner is running along a curve path.

\section{A. the constraints}

To generate forward locomotion, the relative angle $\alpha_{k}$ between the leg and the COM velocity $\mathbf{v}_{k}$ should be bounded within the interval $(0, \pi / 2)$. Figure 4 illustrates the possible $\mathbf{q}_{k}$ that can be produced by changing $\alpha_{k}$ for a right stance when $\eta_{k}$ and $b_{k}$ are held constant. The parameters for the plotted LLS model are $m=2.5 \mathrm{~g}, v_{k}=0.2 \mathrm{~m} / \mathrm{s}, \eta_{k}=1.7 \mathrm{~cm}$ and $b_{k}=1.05 \mathrm{~N} / \mathrm{m}$ which are typical for a cockroach. When $\alpha_{k}=0$ and $\alpha_{k}=\pi / 2$, we have $q_{k}=0$. Therefore, in order to move forward effectively, the angle $\alpha_{k}$ must be within an interval $\left[\alpha_{\min }, \alpha_{\max }\right]$ with $\alpha_{\min }>0$ and $\alpha_{\max }<\pi / 2$. The solid segment in Figure 4 illustrates the possible $\mathbf{q}_{k}$ between $\alpha_{\min }=\pi / 6$ and $\alpha_{\max }=\pi / 3$. There the maximum $q_{k}$ is $1.44 \mathrm{~cm}$. When $\alpha_{k}$ is within $[\pi / 6, \pi / 3]$, the minimum $q_{k}$ is $1.24 \mathrm{~cm}$. The changes in $q_{k}$ is not big for a wide range of $\alpha_{k}$. This is typical for LLS models.

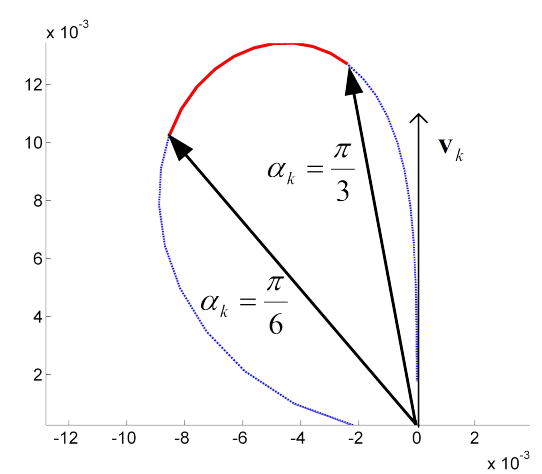

Fig. 4. The possible $\mathbf{q}_{k}$ generated by a right stance for an LLS model $\mathbf{v}_{k}$ is the velocity of the COM. The units are in meters. The curve (dotted and solid) illustrates the end points for vector $\mathbf{q}_{k}$ starting from the origin when $\alpha_{k}$ changes from 0 to $\pi / 2$ while other parameters are constant. The solid segment corresponds to $\alpha_{k} \in[\pi / 6, \pi / 3]$.

The above example suggests that it is possible to keep $\eta_{k}$, $q_{k}$ and $\phi_{k}$ constant for each stance. We control the spring constant $b_{k}$ and the leg placement angle $\alpha_{k}$. The advantage of this strategy is that the distance traveled by the COM is identical for every stance. This fact can help us analyze the tracking behavior later. For the LLS model plotted in Figure 4 , in order to keep $q_{k}=1.44 \mathrm{~cm}$, we plot the spring constant $b_{k}$ as a function of the leg placement angle $\alpha_{k}$ in Figure 5. For $\alpha_{k} \in[\pi / 6, \pi / 3], b_{k}$ lies between $0.78 \mathrm{~N} / \mathrm{m}$ and $1.06 \mathrm{~N} / \mathrm{m}$. This range is not difficult to implement.

With $q_{k}$ constant for each stance, the possible movement of the COM can be depicted by a cone $\mathcal{C}_{k}$. The two edges of the cone correspond to $\alpha_{k}=\alpha_{\min }$ and $\alpha_{k}=\alpha_{\max }$. The length of both edges are $q_{k}$. Figure 6 illustrates cone $\mathcal{C}_{k+1}$ and cone $\mathcal{C}_{k} \cdot \mathcal{C}_{k+1}$ grows from the end point of $\mathbf{q}_{k}$ which lies in the circular arc of $\mathcal{C}_{k}$. We found that $\mathcal{C}_{k+1}$ is the mirror image of $\mathcal{C}_{k}$ with $\mathbf{q}_{k}$ being the axis of symmetry. This is because the velocity vector $\mathbf{v}_{k+1}$ and $\mathbf{v}_{k}$ are symmetric with respect to $\mathbf{q}_{k}$, which can be proved by solving equations (16). Therefore, as the runner moves forward, the cone will flip from side to side. 


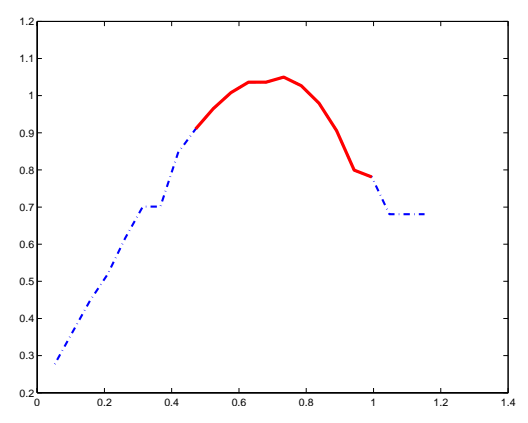

Fig. 5. The spring constant $b_{k}$ (in $\mathrm{N} / \mathrm{m}$ ) as a function of leg placement angle $\alpha_{k}$ (in radians) to keep $q_{k}=1.44 \mathrm{~cm}$. The solid segment corresponds to $\alpha_{k} \in[\pi / 6, \pi / 3]$.

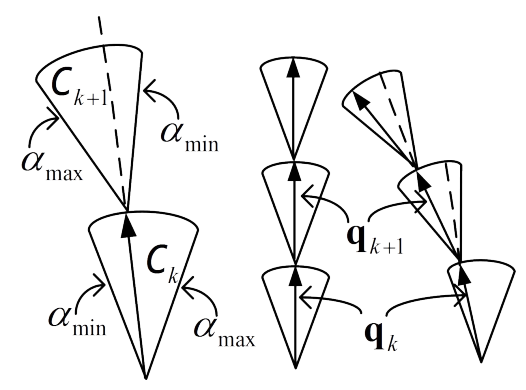

Fig. 6. The cones flip from one side to another when running. On the left, the cones $\mathcal{C}_{k}$ and $\mathcal{C}_{k+1}$ are symmetric with respect to $\mathbf{q}_{k}$ which is the solid arrow. In the middle, the runner is running along a straight line. On the right, the runner is running along a curve path in the counter clockwise direction.

\section{B. running along a curve path and robustness}

We use the index $i$ for all right and left stances. When running parallel to a desired curved path, the COM movement satisfies $\rho_{i}=\rho_{c}$ for all $i$. Therefore, we have $f_{i}=0$ in equation (8). The following conditions are necessary:

A1) $\mathbf{q}_{i} \in \mathcal{C}_{i}$;

A2) $\theta_{i}=\sin ^{-1}\left(q_{i} / 2 \lambda_{i}\right)$.

Condition A1 requires that the COM movement belongs to the cone that is feasible for the constrained model. Condition A2 requires that $\mathbf{q}_{i}$ is parallel to the desired curve.

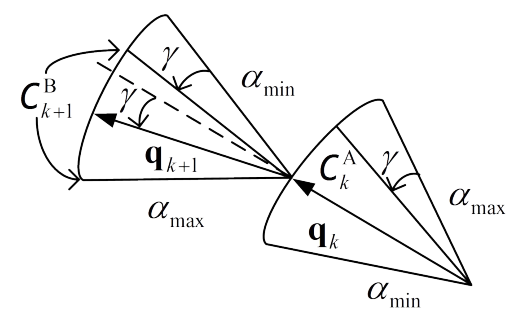

Fig. 7. The sub-cones $\mathcal{C}_{k}^{\mathrm{A}}$ and $\mathcal{C}_{k+1}^{\mathrm{B}} \cdot \mathbf{q}_{k}$ is the middle line of $\mathcal{C}_{k}^{\mathrm{A}}$, and $\mathbf{q}_{k+1}$ is the middle line of $\mathcal{C}_{k}^{\mathrm{A}}$. The angle difference between $\mathbf{q}_{k+1}$ and $\mathbf{q}_{k}$ is $\gamma$.

If the desired curve is a straight line segment, then $\theta_{i}=0$ in condition A2. As the runner moving forward, the cone $\mathcal{C}_{i}$ will be flipping side to side with respect to the straight line. In this case we have $\alpha_{i}=\alpha_{i+1}$. The robustness of this behavior is determined by the size of the cone and the value of $\alpha_{i}$. If we choose the value of $\alpha_{i}$ so that the COM movement $\mathbf{q}_{k}$ is always in the middle of the cone, then the tracking behavior is the most robust. See the middle figure in Figure 6.

If the desired curve is convex with positive curvature, then $\theta_{i} \neq 0$. To find out $\theta_{i}$, we study the $k$ th and $(k+1)$ th stance, i.e., a right stance followed by a left stance. For convenience we let

$$
\gamma_{k}=2 \sin ^{-1} \frac{q_{k}}{2 \lambda_{k}}
$$

Observe from Figure 2 that

$$
\theta_{k+1}-\theta_{k}=-\left(\alpha_{k+1}-\alpha_{k}\right)+\gamma_{k}
$$

Condition A2 implies that $\theta_{k+1}=\theta_{k}=\gamma_{k} / 2$. Therefore, when running along a convex curve, $\alpha_{k+1}-\alpha_{k}=\gamma_{k}$ should be satisfied. We have similar relation for stance $k+1$ and $k+2$, i.e., a left stance followed by a right stance: $\alpha_{k+2}-$ $\alpha_{k+1}=-\gamma_{k+1}$. We can then write $\alpha_{i+1}-\alpha_{i}= \pm \gamma_{i}$ for all stances. This equation requires that $\gamma_{i}$ must be less than $\left(\alpha_{\max }-\alpha_{\min }\right)$. This implies that $\lambda_{i}$, the instantaneous radius of the curve must satisfy

$$
\lambda_{i}>\frac{q_{i}}{2 \sin \frac{\alpha_{\max }-\alpha_{\min }}{2}} .
$$

This condition is stricter than $\lambda_{i}>q_{i} / 2$ required by Lemma 3.2. Hence the constraint on $\alpha_{i}$ puts a tighter restriction on the curvature of the curve that can be traced.

We divide $\mathcal{C}_{i}$ into two sub-cones. Let $\mathcal{C}_{i}^{\mathrm{A}}$ be the cone for $\alpha \in\left[\alpha_{\min }, \alpha_{\max }-\gamma_{i}\right]$. Let $\mathcal{C}_{i}^{\mathrm{B}}$ be the cone for $\alpha \in$ $\left[\alpha_{\min }+\gamma_{i}, \alpha_{\max }\right]$. Because $\mathcal{C}_{i}$ flips and $\alpha_{i+1}-\alpha_{i}= \pm \gamma_{i}$, if $\mathbf{q}_{i}$ belongs to $\mathcal{C}_{i}^{A}$, then $\mathbf{q}_{i+1}$ belongs to $\mathcal{C}_{i+1}^{\mathrm{B}}$. Now consider a right stance $k$ followed by a left stance $(k+1)$. When running along a convex curve in the counter clockwise (CCW) direction, the runner must have $\alpha_{k} \in \mathcal{C}_{k}^{\mathrm{A}}$ and $\alpha_{k+1} \in \mathcal{C}_{k+1}^{\mathrm{B}}$, see Figure 7. When running in the clockwise (CW) direction, the runner must have $\alpha_{k} \in \mathcal{C}_{k}^{\mathrm{B}}$ and $\alpha_{k+1} \in \mathcal{C}_{k+1}^{\mathrm{A}}$.

To increase the robustness of the tracking behavior, we should let the COM movement $\mathbf{q}_{i}$ be close to the middle of $\mathcal{C}_{i}$. In the case of convex curves, the best choice is to let $\mathbf{q}_{i}$ and $\mathbf{q}_{i+1}$ be symmetric with respect to the middle line of $\mathcal{C}_{i}$. The middle lines of $\mathcal{C}_{i}^{\mathrm{A}}$ and $\mathcal{C}_{i}^{\mathrm{B}}$ are symmetric with respect to the middle line of $\mathcal{C}_{i}$. The angle between the middle lines of $\mathcal{C}_{i}^{\mathrm{A}}$ and $\mathcal{C}_{i}^{\mathrm{B}}$ is $\gamma_{i}$. If the curve has constant curvature, then $\gamma_{i}$ is constant for all $i$. This implies that the middle lines of $\mathcal{C}_{i}^{\mathrm{A}}$ and $\mathcal{C}_{i+1}^{\mathrm{B}}$ are symmetric with respect to the middle line of $\mathcal{C}_{i}$. Therefore, we may choose $\mathbf{q}_{i}$ to be the middle line of either $\mathcal{C}_{i}^{\mathrm{A}}$ or $\mathcal{C}_{i}^{\mathrm{B}}$ for maximum robustness. If the curve has a changing positive curvature, then $\gamma_{i}$ can be different from stance to stance. This "middle line" strategy can not be enforced. In this case one can choose $\mathbf{q}_{i}$ to be as close to the middle lines as possible.

If the desired curve is not convex, the tracking behavior can be viewed as switching between tracking a locally convex curve in the $\mathrm{CCW}$ direction and in the $\mathrm{CW}$ direction. The switching depends on how the curve changes from locally convex to locally concave. No general conclusions can be drawn regarding which part of the cone $\mathcal{C}_{i}$ is used 
for a stance. In this case the tracking behavior is not a "steady state".

\section{Simulations}

We present simulation results to demonstrate tracking a circle centered at the origin with radius $0.02 \mathrm{~m}$. The parameters for the LLS model are the same as in section V. The desired distance to the circle is $0.03 \mathrm{~m}$. Initially, the runner start from $(0.1,0)$ outside the circle. The speed of the COM is $0.2 \mathrm{~m} / \mathrm{s}$. The initial direction of the velocity is $\pi / 3$. The leg placement angle $\alpha_{i}$ are constrained to be within the interval $(\pi / 6, \pi / 3)$. We change the spring constant $b_{k}$ so that $q_{i}$ is always equal to $1.53 \mathrm{~cm}$, which is $90 \%$ of the leg length at rest.The gain $K_{i}$ is selected to be 0.5 . When $\theta_{i}$ can not be achieved by $\alpha_{i}$, we simply use the value for $\alpha_{i}$ that will minimize the differences between desired $\tilde{\theta}_{i}$ and achievable $\theta_{i}$; hence implemented the approximation method.

The trajectory of the COM and the distance between the COM and the circle are plotted in Figure 8 . We see that the convergence is achieved after 12 stances which take less than one second.

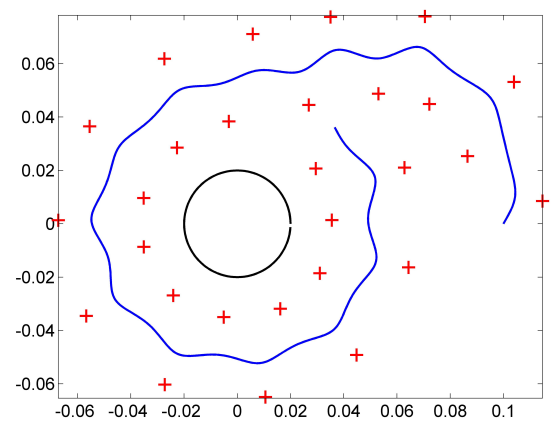

(a) trajectory of COM

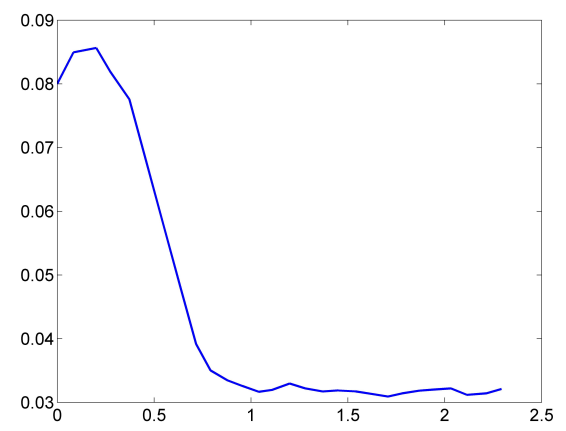

(b) distance between COM and the circle

Fig. 8. Tracking a circle with radius $0.02 m$. In (a), the cross symbols indicate the touchdown points. The units for both horizontal and vertical axis are meters. In (b), the distance (in meters) between the COM and the circle is plotted as a function of time (in seconds). We can see it converges to the desired separation $0.03 m$.

\section{SUMMARY AND FUTURE WORK}

We have analyzed the control of LLS model and designed a hybrid curve tracking control law for legged locomotion.
Using measurements of the curve for feedback, the discrete algorithm guarantees convergence to the desired curve path. During each stance, the controlled continuous dynamics is analyzed. The parameters of the LLS model is determined to implement the discrete algorithm at the beginning of each stance. We have also investigated the effects of parameter constraints. These constraints limited tracking ability. For straight lines and convex curves, a steady state can be reached. The robustness of these steady states depends on the range for the parameters.

Interesting results regarding wall following behaviors of cockroaches are reported by Camhi and Johnson in [11]. Recently, a wall following wheeled robot using antenna like tactile sensor was reported in [8]; curve tracking for atomic force microscope is discussed in [12]; a general boundary tracking control law is derived for Newtonian particles in [13]. Our work, although intended for legged locomotion, may be adapted to handle other cases, for example, locomotions with flapping wings [14].

\section{REFERENCES}

[1] R. Full and D. E. Koditschek, "Templates and anchors: Neuralmechanical hypothesis of legged locomotion on land," Journal of Experimental Biology, vol. 83, pp. 3325-3332, 1999.

[2] J. Schmitt and P. Holmes, "Mechanical models for insect locomotion: Dynamics and stability in the horizontal plane I. Theory," Biological Cybernetics, vol. 83, pp. 501-515, 2000.

[3] — "Mechanical models for insect locomotion: Dynamics and stability in the horizontal plane II. Applications," Biological Cybernetics, vol. 83, pp. 517-527, 2000.

[4] — "Mechanical models for insect locomotion: Stability and parameter studies," Physica D, vol. 156, pp. 139-168, 2001.

[5] J. Schmitt, M. Garcia, R. Razo, P. Holmes, and R. J. Full, "Dynamics and stability of legged locomotion in the horizonatoal plane: A test case using insects," Biological Cybernetics, vol. 86, pp. 343-353, 2002.

[6] R. M. Ghigliazza, R. Altendorfer, P. Holmes, and D. Koditschek, "A simply stablized running model," SIAM Review, vol. 47, no. 3, pp. 519-549, 2005.

[7] F. Zhang, A. O'Connor, D. Luebke, and P. S. Krishnaprasad, "Experimental study of curvature-based control laws for obstacle avoidance," in Proc. 2004 IEEE International Conf. on Robotics and Automation, New Orleans, LA, 2004, pp. 3849-3854.

[8] A. G. Lamperski, O. Y. Loh, B. L. Kutscher, and N. J. Cowan, "Dynamical wall-following for a wheeled robot using a passive tactile sensor," in Proc. 2005 IEEE International Conf. on Robotics and Automation, Barcelona, Spain, 2005, pp. 3838-3843.

[9] V. Arnold, Mathematical Methods of Classical Mechanics 2nd Ed. New York: Springer, 1989.

[10] D. Jindrich and R. J. Full, "Many legged maneuverability: dynamics of turning in hexapods," Journal on Experimental Biology, vol. 202, pp. 1603-1623, 1999.

[11] J. M. Camhi and E. N. Johnson, "High-frequency steering maneuvers mediated by tactile cues: Antennal wall-following in the cockroach," Journal of Experimental Biology, vol. 202, pp. 631-643, 1999.

[12] S. B. Andersson and J. Park, "Tip steering for fast imaging in AFM," in Proc. 2005 American Control Conf., Portland, OR, June 6-10, 2005, pp. 2469-2474.

[13] F. Zhang, E. Justh, and P. S. Krishnaprasad, "Boundary following using gyroscopic control," in Proc. 43rd IEEE Conf. on Decision and Control, Atlantis, Paradise Island, Bahamas, 2004, pp. 5204-5209.

[14] S. D. Ross, "Optimal flapping strokes for self-propulsion in a perfect fluid," in Proc. 2006 American Control Conf. Minneapolis, MN: IEEE, 2006, pp. 4118-4122. 